\title{
Un sujet marginal: les communautés LGBTQ assimilées à de boucs émissaires dans le film 120 battements par minute
}

\author{
${ }^{1}$ Rosidin Ali SYABANA, ${ }^{2}$ Wening UDASMORO \\ 1,2 Universitas Gadjah Mada - Indonésie
}

Reçu le 30 octobre 2019 | Accepté le 31 décembre 2019

RÉSUMÉ. Cet article porte un regard sur le grand nombre de films allosexuels produits par les pays occidentaux qui donnent une visibilité aux communautés homosexuelles, bisexuelle, transgenre, transsexuelle ou queer (LGBTQ). Cependant, elles restent marginalisées. Considérées comme des parasites qui renversent des valeurs ancestrales conventionnelles, les communautés LGBTQ servent de boucs émissaires pour endosser la responsabilité de la propagation du VIH/SIDA et de cette crise de l'épidémie. Le film 120 Battements par Minute réalisé par Robin Campillo est un film qui traite du rôle et du bouc émissaire incarné par les groupes LGBTQ. Cet article utilise la théorie du bouc émissaire par Girard (1982) en révélant que dans une société, il existe toujours un groupe sacrifié stigmatisé en temps de crise. Cet article utilise l'analyse du discours multimodal de Gunther Kress et Theo Van Leewuen (2004) pour analyser des extraits du film. La conclusion montre que le système de bouc émissaire utilise des stéréotypes et des préjugés en identifiant un groupe qui est proche de la crise pour ensuite le nommer en tant que victime. Puisque les premiers cas détectés de patients infectés par VIH/SIDA sont issus de communautés LGBTQ, elles sont donc considérées comme responsables de la crise.

Mots-clés : analyse du discours multimodal, bouc émissaire, film allosexuel, VIH/SIDA.

\begin{abstract}
This article explores how LGBTQ people in the West use film as a space for narrating themselves. LGBTQ people remain marginalized, being stigmatized as parasites who disrupt the established socio-cultural order and blamed for HIV/AIDS pandemic. Robin Campillo's film 120 Battements par Minute (120 Beats per Minute) deals specifically with how the LGBTQ community has been scapegoated. Girard argues that, when a crisis occurs, a social group must be sacrificed during a crisis occurs in order to resolve it. For its analysis, this article applies the multimodal discourse approach proposed by Gunther Kress and Theo Van Leewuen to images and still frames from the film, finding that the LGBTQ community has been scapegoated through stereotypes and prejudices. As they have been popularly identified with the HIV/AIDS crisis, members of the LGBTQ community have been blamed - and expected to take responsibility - for it.
\end{abstract}

Keywords: Multimodal Discourse analysis, scapegoat, LGBT film, HIVIAIDS.

囷 auteur correspondant : rosidinali421@gmail.com

Pour citer cet article (Style APA) : Syabana, R.A. \& Udasmoro, W. (2019). Un sujet marginal: les communautés LGBTQ assimilées à de boucs émissaires dans le film 120 battements par minute Francisola: Revue Indonésienne de la langue et la littérature françaises, 4(2), 106-114. doi: 10.17509/francisola.v4i2.24201. 


\section{INTRODUCTION}

$\mathrm{Au}$ cours des dernières décennies, la réalisation et la production de films allosexuels se sont accrues en Occident. L'objectif est de partager, à travers la fiction, des histoires de LGBTQ. Pour ce faire, le film est utilisé comme un medium (Rich, 2000) afin de donner aux communautés LGBTQ la possibilité de présenter au public sur des sujets qu'ils n'avaient jamais abordé en raison de la discrimination qu'ils subissent depuis longtemps (Balsam et al, 2011, p.164). Cette plateforme est également un moyen pour tisser un dialogue entre les communautés LGBTQ et les autres segments de la société et fournir des informations sur la pluralité d'identités sexuelles, car elles ne l'étaient pas auparavant, ne disposant pas d'une plateforme massive, i.e. un film, pour signifier leur existence (Czach, 2004, p.85).

En Occident, le film allosexuel offre un nouvel espace à l'industrie cinématographique pour décrire les communautés LGBTQ. Il illustre beaucoup de thématiques, y compris la santé, la discrimination et la reconnaissance de son orientation sexuelle et de la situation familiale (Rees-Roberts, 2008). Cependant, il existe peu de film allosexuel qui aborde le sujet du VIH/SIDA. Aujourd'hui, les films allosexuels produits se concentrent plutôt sur les relations sexuelles $\mathrm{du}$ couple que sur l'échange de sujet méconnu, i.e. l'épidémie de VIH/sida (Román, 1992, p.321). Il est important que le sujet du VIH/sida fasse l'objet de discussion car?

Cette tendance s'applique également aux films allosexuels en France. Le film allosexuel français a commencé à gagner sa popularité à la fin du XXe siècle avec des films tels que Gazon Maudit (1995), Ma Vie en Rose (1997), Belle Maman (1999) et Le Derrière (1999). L'industrie cinématographique française offre un espace aux communautés LGBTQ pour raconter leur coming-out en tant que allosexuels vers médias traditionnels (Johnston, 2002, p.24). Puis, au début du XXIe siècle, les films allosexuels ont commencé à aborder les problèmes de discrimination et d'ethnicité, comme dans le film Drôle de Felix (2000), qui lance un nouveau débat de société (Provencher, 2008, p. 52-56). On relève une évolution dans la dynamique des récits de film allosexuel en France en passant du stade de reconnaissance d'une communauté au soulèvement de problématiques (discrimination, ethnicité)

Certains des films mentionnés soulèvent des problèmes du VIH/SIDA. Cependant, la manière dont ces films traitent $\mathrm{du} \mathrm{VIH} /$ sida ne fait qu'introduire le personnage principal du récit en tant que séropositif, comme si le sujet même du VIH/sida n'était pas central et ne méritait pas de pousser plus loin la réflexion, comme dans le film Drôle de Félix (2000) (Provencher, 2008, p. 53). Pourtant, il semble important d'échanger sur le sujet principal du film: la conscience de la société aux pratiques sexuelles non-hétéro normatives et sexuelles (Müller, 2017, p.2). Le film, en tant que un mass média et le médium le plus accessible à la société, il véhicule des messages.

120 Battements par Minute (2017) de Robin Campillo est l'une des œuvres de fiction dramatique portant sur le VIH/SIDA. Ce film raconte l'histoire d'un groupe d'activistes homosexuels issu d'ACT UP (Association de lutte contre le SIDA). Ces militants s'activent pour lutter contre l'indifférence et demandent au gouvernement des campagnes d'information. La plupart des séquences du film sont dominées par les manifestations d'ACT UP contre les entreprises pharmaceutiques, le gouvernement et pour la sensibilisation dans les établissements scolaires. Bien que certains de leurs membres soient morts lors des manifestations et $d^{\prime}$ actions militantes, les résultats sont moindres.

Ce film offre un nouvel espace de dialogue et une nouvelle lecture pour les communautés LGBTQ ainsi que pour la société. Car le film soulève la lutte du groupe LGBTQ contre le VIH/SIDA de manière plus profonde que les films allosexuels précédents. Cependant, ce film semble accuser la communauté LGBTQ d'être la source de transmission du VIH/sida. Pourtant, la responsabilité de cette épidémie ne peut pas être entièrement imputée aux communautés 
LGBTQ. Cela consiste à déplacer la faute sur les groupes LGBTQ. Ce système de déplacement est nommé bouc émissaire par René Girard, l'imputation d'une problématique à un groupe marginalisé (Girard, 1982,p. 3).

Le système de bouc émissaire existe car un certain groupe a envisagé de changer les normes culturelles anciennes mis en péril pour la paix dans la société. Les boucs émissaires sont un moyen de libérer la société d'une crise menaçant des normes construites, décrites par le sociologue Tom Douglas:

La procédure du bouc émissaire était essentiellement un processus de purification, ce qui signifie en substance que ses praticiens ont le sentiment d'être contaminés par les contraventions de normes traditionnelles et que c'est un moyen efficace pour rétablir les normes précédentes dans les yeux de la société, et plus important, dans ceux de leur Dieu (Douglas, 2003, p.14).

D'après la citation ci-dessus, on peut constater que le rôle du bouc émissaire est d'éradiquer les crises survenant dans la société en espérant que la norme ancienne puisse revenir sans aucune nouvelle norme destructive. Dans le film 120 Battements par Minute (2017), le groupe LGBTQ est considéré comme la partie destructive de la société à la norme usuelle, à savoir à la valeur traditionnelle de non-hétérosexuelles qui est attachée depuis longtemps. Cependant, est-ce seulement le facteur de valeur qui justifie leur identité de bouc émissaire dans la narration $\mathrm{du}$ film? Y a-t-il d'autres raisons pour lesquelles les groupes LGBTQ sont accusés comme source de crise dans la société et se positionne en tant qu'un bouc émissaire? Cet article traitera de la justification du film 120 Battements par Minute (2017) en plaçant les groupes LGBTQ comme des boucs émissaires dans la lutte qu'ils mènent pour exhorter le gouvernement pour résoudre le problème d'épidémie du VIH/sida.

Les articles sur les boucs émissaires dans les œuvres littéraires ont déjà été rédigés. Alan Bernard McGill (2018) décrit le phénomène de bouc émissaire dans le film The Witch (2015) et l'article de David Humbert (2010) en analysant le système du bouc émissaire dans le film Les Oiseaux (1963). En outre, il existe également des œuvres littéraires qui ont fait l'objet de recherche utilisant la théorie de bouc émissaire de René Girard, par exemple l'article Isaac Slater (2012) qui parle d'une nonne violée à la vue du bouc émissaire et l'article de Christopher P. Atwood (2009) qui aborde le sacrifice de frères dans l'histoire mongole. Au-delà de cela, dans les divers articles mentionnés, aucun n'a parlé du système de bouc émissaire $\mathrm{du}$ groupe LGBTQ. La plupart des articles existants se concentrent plutôt sur les romans ou films religieux. Cet article offre une nouvelle perspective sur le phénomène du bouc émissaire attribué aux groupes marginalisés ayant des orientations sexuelles différentes.

\section{MÉTHODE}

La méthode utilisée dans cet article est la méthode d'analyse du discours multimodal de Theo van Leeuwen et Gunther Kress (2004) qui est l'une des caractéristiques de la sémiotique sociale. La sémiotique sociale est une branche de la sémiotique qui constate que le signe est une chose dynamique (Leeuwen, 2004, p.17). Le signe en sémiotique sociale est considéré comme un outil pour montrer le discours à délivrer au public, à la fois en termes d'histoire, culture sociale, ainsi que de son utilisation potentielle à l'avenir. En sémiotique sociale, le signe n'est pas seulement constitué d'éléments linguistiques, mais également d'éléments visuels, de couleurs, de musique, d'expressions faciales, de continuité et de discontinuité et d'autres éléments.

L'object analysé dans cette recherche est le film 120 Battements par Minute réalisé par Robin Campillo. Les éléments linguistiques, ainsi que d'autres signes, surtout les visuels sont collectionnés en tant que corpus des donnés. Les unités d'analyse sont des aspects linguistiques tels que les mots, et les phrases trouvés dans les scènes $\mathrm{du}$ film. Les signes visuels liés aux problèmes de marginalisations, d'exploitations et de dominations sont aussi considérés comme les donnés importants. Les aspects tirés sont basés sur le problème apparu dans le film (le 
bouc émissaire). L'analysé des donnés regard surtout la position des groups ou des personnes de LGBTQ dans le film.

Comme procédure, premièrement les scènes avec les problèmes liés au bouc émissaire sont collectionnées. Deuxièmement, les aspects linguistiques, tels que les mots et les phrases et les aspects visuels sont classifiés. Troisièmement, les même modèles des unités sont mis dans la même calssification. Les choix des unités linguistiques ainsi que visuels sont analysés en utilisant les concept de bouc émissaire de René Girard.

\section{RÉSULTATS ET DISCUSSION}

\subsection{Les concepts sur les relations entre} LGBTQ et le HIV/SIDA perçus bouc émissaire

Dans son livre Le Bouc émissaire (1982), Girard exploite le terme stéréotype de persecution pour décrire le processus de positionnement du bouc émissaire dans la société. La phase initiale de ce processus consiste à identifier la crise et à sélectionner la partie qui décrit les caractéristiques les plus proches de la crise. En effet, quand une société est sur un pied d'égalité et qu'il y a une nouvelle valeur considérée comme étrangère, il y aura une relation réciproque (Girard, 1982,p. 21). Cette relation réciproque engendre des différences dans la société et donne lieu à des boucs émissaires.

La relation réciproque se découvre dans le film 120 Battements par Minute (2017) lorsque l'épidémie de HIV/SIDA devient la crise principale dans l'histoire. Les compagnies pharmaceutiques font des compromis positifs, par exemple, sur la base de scénarios de films, de conférences mondiales sur le HIV/SIDA, de recherches sur les vaccins et les pilules pour prévenir la propagation $\mathrm{du}$ virus dans le corps et d'autres méthodes. Cependant, il existe également une réponse négative au HIV/SIDA qui blâme les groupes LGBTQ ce qui entraîne des accusations négatives à leur encontre.

Un exemple tiré du film: lorsque le groupe ACT UP a publié une affiche sur l'événement Gay Pride, un festival du groupes LGBTQ afin de célébrer leur identité sexuelle dans les espaces publics. Un jeune homme les a accusés d'être la source de la propagation du virus HIV/SIDA.

Le mec: Eh Putain! Pourquoi tu ne nous laisse pas tranquille? On ne fait rien mal et on prend notre temps à se promener et vous tous ce que vous trouvez à faire c'est nous faire peur avec vos affiches. (00:34:21).

D'après la citation ci-dessus, il est à noter que certains termes indiquent que le jeune homme a reproché au groupe LGBTQ de diffuser la communication du VIH/SIDA. Le jeune homme a affirmé que l'épidémie de HIV/SIDA provoque des menaces et des craintes sur la communauté et qu'il avait fait cette déclaration devant le groupe LGBTQ. Le jeune homme a explicitement reproché la cause de cette publicité sur l'épidémie au groupe LGBTQ parce qu'il présumait que leur vie était normale avant l'épidémie de HIV/SIDA. Outre les termes textuels, il positionne les groupes LGBTQ comme bouc émissaire vis-à-vis à des groupes LGBTQ dans la transmission du VIH/SIDA comme dans le découpage de l'image ci-dessous:

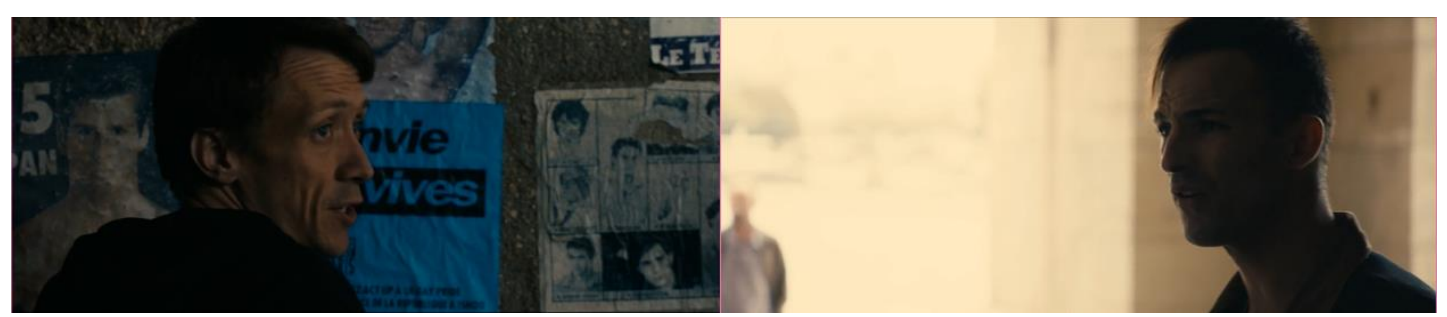

Image 1. Les membres d'ACT UP (à gauche) expliquent à un jeune homme (à droite) leurs activités de défense des droits de l'homme affectés par le VIH/ SIDA. 
Du point de vue du jeune homme (à droite), il sourcille et arbore une expression contrainte envers les membres d'ACT UP (à gauche). Cette expression témoigne de son désaccord face aux actions d'ACT UP. Ce comportement est complété par l'utilisation d'un ton virulent dans son discours renforçant le caractère réprobateur des actions d'ACT UP.

La culpabilité et la dénonciation des groupes LGPTQ sont liées à la peur et l'intolérance. Lorsqu'il 'apparait une crise au sein d'une communauté, les membres de la communauté préfèrent affecter la crise à un autre groupe, étrange ou marginal, au lieu de désigner le vrai responsable. C'est un moyen plus simple et plus rapide pour résoudre un problème (Girard, 1982,p. 22). En outre, la communauté hétérosexuelle est apeurée à l'arrivée des nouvelles valeurs (non issues du schéma hétérosexuel) susceptibles de modifier l'ordre de la société. Les communautés hétérosexuelles ont peur d'être infectées par le HIV/SIDA en s'entourant du groupe LGBTQ, de sorte que le concept selon lequel le groupe LGBTQ est la principale source de propagation du HIV/SIDA et finalement le terme d'homophobie apparaît.

De plus, sa nature minoritaire et marginale est également l'un des facteurs aggravant pour les groupes LGBTQ. Girard (1982, p.25) avoir une opinion sur le système de victimisation, dénoncé arbitrairement, par exemple des groupes religieux ou ethniques qui constituaient une minorité dans la communauté. ACT UP, dont les membres sont principalement originaires des groupes LGBTQ, est composé de minorités qui essaient de trouver des organisations ou des clubs pour échanger des idées. Cependant, quand ils livrent leurs opinions en public, ils sont exécutés ou punis par les communautés hétérosexuelles et les forces de l'ordre.

L'image ci-dessus démontre comment le groupe LGBTQ est perçu comme un groupe minoritaire. ACT UP ne compte que deux membres qui sont encerclés par la police. En outre, la police a également utilisé un équipement de protection complet uniquement pour arrêter la manifestation d'ACT UP. La photo ci-dessus montre un groupe minoritaire spolié, même s'il défend les personnes affectées par HIV/SIDA.

La discrimination à l'encontre des groupes musulmans minoritaires en Inde et au Myanmar est un autre exemple de persécution des minorités dans le système des boucs émissaires. Le cas du pays cité peut être considéré du point de vue du bouc émissaire en reliant le caractère minoritaire du groupe à la crise actuelle. Selon la parole de Douglas (2003, p.54),

Few, if any, of these early multi-ethnic communities were democratic organisations and the problems generated by ethnic minorities or peoples with different religions could be treated with either summary brutality or easy tolerance.

Peu de ces premières communautés multiethniques, voire aucune, n'étaient des organisations démocratiques et les problèmes engendrés par les minorités ethniques ou les peuples de religions différentes pourraient être menacés soit par une brutalité sommaire ou bonne tolérance. (p.54)

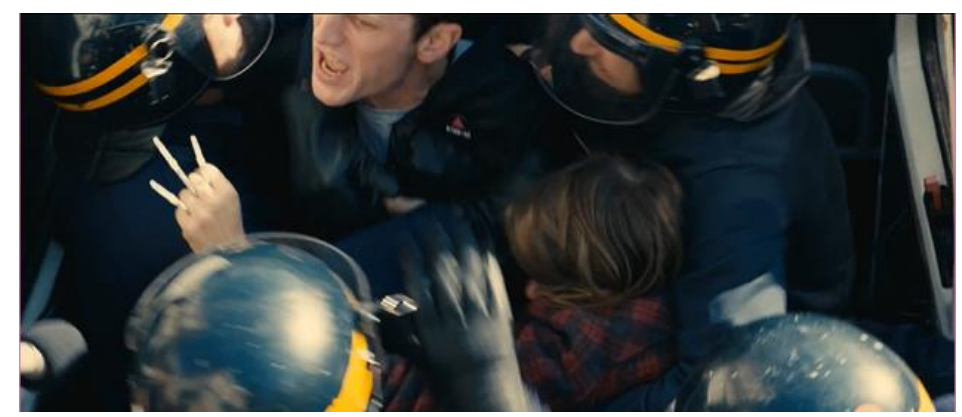

Image 2. Persécution d'ACT UP par la police pendant la manifestation 
Cependant, parfois, la communauté choisit la persécution directe en partant du principe qu'en exécutant les victimes, la source de la crise va bientôt disparaître et l'ordre communautaire établi de longue date va revenir à la normale sans aucune valeur extérieure jugée menaçante. C'est ce que Girard appelle une crise du cadavre, à savoir qu'une crise nécessitera toujours des victimes dans tous les conflits et que la communauté utilise un système d'unanimité pour la sélection des victimes (Shindhunata, 2007, p.128).

Comme on le voit dans le film, le groupe LGBTQ est devenu une victime du système universel. La communauté hétérosexuelle les a accusés d'être responsables de l'épidémie de VIH/SIDA et la police a également contribué à la persécution des victimes. Enfin, la relation entre les groupes LGBTQ et la propagation du HIV/SIDA ne repose que sur l'accusation de liens qui unissent les uns aux autres, de relations sexuelles libres et, de ce fait, le HIV/SIDA se propage facilement bien que tout le monde ait les mêmes chances d'être infecté par le HIV/SIDA si le partenaire sexuel est séropositif (séropositif). Dans le film 120 Battements par Minute (2017), le groupe LGBTQ semble être infecté par le VIH/SIDA, de sorte qu'il est devenu la cible de la persécution de la communauté hétérosexuelle.

\subsection{Activisme et anarchisme: images négatives de groupes LGBTQ}

Dans le film 120 Battements par Minute (2017), en plus d'être accusés de relations entre les groupes LGBTQ et le HIV/SIDA, les groupes LGBTQ sont considérés coupables de leur anarchisme lorsqu'ils protestent contre les cartels de la drogue ou le gouvernement.
Les actions anarchistes sont amplifiées par les histoires pour rendre l'image du groupe LGBTQ négative devant le public, même si leur objectif de la manifestation était de faire entendre la voix d'un groupe minoritaire.

Comme par exemple, lorsque ACT UP a organisé une manifestation contre l'Agence française de lutte contre le sida (AFLS), ils ont poursuivi cette société en justice parce qu'il n'y avait pas eu de progrès depuis trois ans dans leurs recherches sur les méthodes de traitement des personnes affectés de HIV/SIDA.

Le groupe ACT UP accuse et s'interroge sur la pertinence de l'AFLS en tant qu'institution qui développe la méthodologie du traitement du VIH/SIDA. Malgré quelques années d'existence, ACT UP ne mesure pas l'impact de l'AFLS. Une des membres de l'ACT UP qui s'appelle Sophie a même rappelé dans son discours que la France était le pays européen enregistrant à cette époque le nombre plus important de séropositifs. Toutefois, leur action est considérée comme un acte anarchiste dans la manifestation d'ACT UP. Ils ont eu une altercation avec le directeur de l'AFLS lors d'une présentation dans une conférence sur le VIH/SIDA.

La photo ci-dessus nous montre à quel point la narration d'un film peut illustrer l'anarchisme du groupe LGBTQ. Les membres d'ACT UP (à droite et derrière) ont tiré le directeur de l'AFLS (devant) et ont montré les expressions de visage comme s'ils l'ont blessé. La couleur sang sur le visage du directeur provient du liquide rouge lancé par d'autre membre lors du discours du directeur. Cette fiction est tournée comme si le groupe LGBTQ était instigateur de la violence et avait torturé le directeur en public.

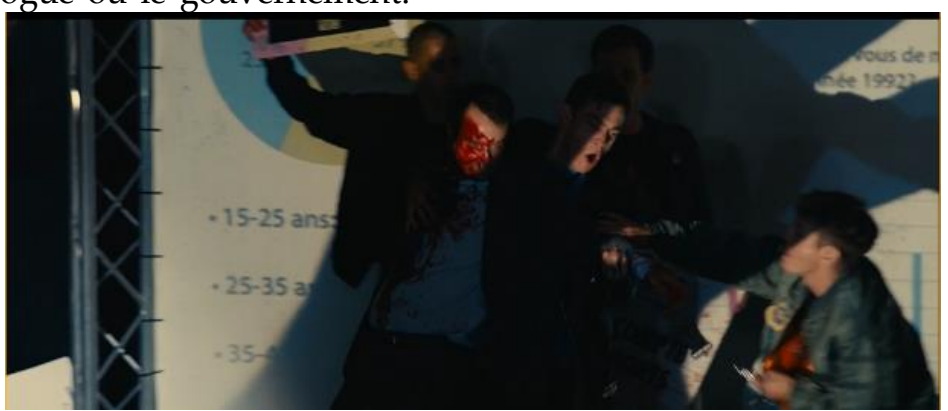

Image 3. Altercation entre le directeur de l'AFLS et les membres d'ACT UP 
Le phénomène est né de la distinction entre la société et le groupe LGBTQ. Ceci mène à la différence de perception entre les deux parties et génère une incompréhension (Sindhunata, 2007, p. 139). On voit cette différence dans les manifestations de l'ACT UP utilisant la violence comme montré dans l'image 2 ci-dessus. La société considère que le groupe LGBTQ est un groupe anarchiste, en se référant au discours négatif diffusé (Cf. scène de la manifestation contre l'AFLS). Cette manifestation d'ACT UP a eu lieu fin des années 1980, à une époque où une frange de la société catégorisait le groupe LGBTQ comme un groupe dangereux avec des stéréotypes de VIH/SIDA, consommation de drogues, et autres préjugés.

Pour ces multiples raisons, le groupe LGBTQ est considéré comme un parasite dans la société. Nous observons un regard binaire de l'opposition dans le cas de manifestation contre l'AFLS. Le groupe LGBTQ est un groupe négatif qui pèse sur la vie sociétale, sur le plan de l'orientation sexuelle et de la protestation dans le lieu public. Au contraire, l'AFLS est considéré comme la partie altruiste qui aide les séropositifs. Rien n'est ni tout noir, ni tout blanc bien entendu. Il faudrait connaitre la raison pour laquelle le groupe ACT UP exige de l'AFLS à préciser ses projets. Ce dernier a passé beaucoup de temps à développer le traitement contre le $\mathrm{VIH} / \mathrm{SIDA}$ sans avoir de résultat satisfaisant, en mentionnant une augmentation des cas de séropositivité en France, sans développement de médicament pour arrêter la contagion du virus.

Néanmoins, la société n'a qu'une vision périphérique. Ils n'ont pas d'expertise sur le sujet. La majorité de la population juge les actions d'ACT UP en se basant sur la manifestation contre l'AFLS, et la distinction dans la société. Selon Girard, le bouc émissaire est de caractère minoritaire, facile à devenir victime, et ayant un critère physique anormal comme la maladie, la folie, et cætera.
Il existe des critères strictement physiques qui se rajoutent à la culture et à la religion. La maladie, la folie, la déformation génétique, blessures accidentelles, et même des handicapés en général ont tendance à polariser des persécuteurs. Il suffit juste de regarder autour ou à l'intérieur pour comprendre l'universalité. Même aujourd'hui les gens ne peuvent pas maîtriser un recul instantané face à une anomalie physique (Girard, 1982, p.20).

La citation ci-dessus montre que le groupe LGBTQ dans la fiction 120 Battements par Minute (2017) est devenu bouc émissaire à cause de critère physique. Ceci est prouvé tout au long du film; le caractère anarchiste (attitude dans la société), VIH/SIDA (la maladie), et leur orientation sexuelle (anormalité). La société ne l'évalue que de l'extérieur, sans mesurer des raisons derrière leurs actions. Cette image physique est ensuite répandue dans la société devenue des stéréotypes des LGBTQ sur la place publique.

C'est ainsi que le système de bouc émissaire fonctionne dans la société. Il s'en remet au système de l'unanimité que désigne la victime selon le plus grand nombre et le critère physique qui correspond à la crise dans la société (Sindhunata, 2007). Le groupe LGBTQ est vivement touché par la contagion du VIH/SIDA et observé comme victime dans cette crise. Dans le processus de bous émissaire, la victime va être punie et considérée comme parasite dans la société. Elle menace la structure de la société qui est construite de longue date.

Dans le film 120 Battements par Minute (2017), nous avons trouvé deux punitions pour le groupe LGBTQ. La première est une punition physique administrée par la police. Cette institution est un symbole de la discipline. Dans la fiction, elle punit le groupe LGBTQ durant la manifestation. Cette action est censée apaiser le conflit entre ACT UP et l'autre partie. Nous pouvons voir quelques images de cette scène de la police contre le groupe ACT UP ci dessus. 

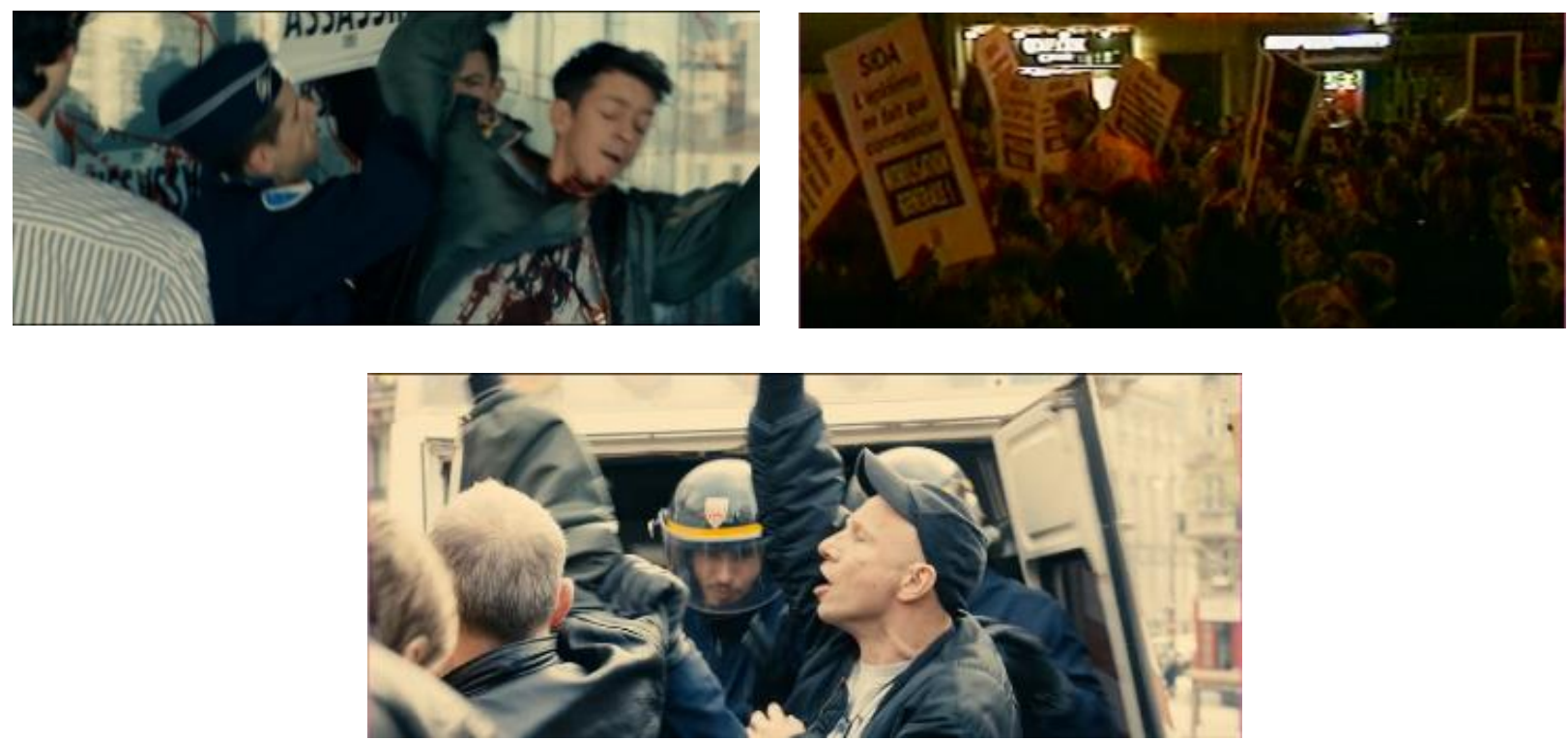

Image 4. Altercation des membres d'ACT UP contre les policiers.

La deuxième sanction est une peine verbale (exp: discours de haine envers le groupe LGBTQ). Comme en témoigne la séquence où ACT UP demande l'état $\mathrm{d}^{\prime}$ avancement de recherche de médicament à l'AFLS, quelques medias français réagissent sur cet évènement. Aides est l'une des associations qui condamnent dans les medias la manifestation d'ACT UP contre l'AFLS comme ci-dessous;

«Aides condamne les actions d'Act Up Paris qui sont physiquement vers Michel Bernin, directeur de l'AFLS Bla... Bla... Bla...»Et attend j'ai vu à la fin, là, voilà, "nous condamnons d'ambiguïté de la méthode $d^{\prime}$ Acte Up à la fois brutale et enfantine même si nous comprenons l'impatience de militants d'Acte Up face à l'inefficacité de l'action publique d'AFLS en particulier (00:11:3700:11:59).

Dans cette citation, Aides utilise des choix de mots qui illustrent que l'ACT UP est un groupe violent et puéril, L'usage des mots brutal et infantile indique qu' Aides n'adhère pas à l'action de manifestation d'ACT UP. Les messages négatifs sont finalement délivrés à la société quand le public reçoit la nouvelle. Ces journalistes colportent une image négative d'ACT UP et conditionne une perception de violence et de brutalité de la société à l'égard des groupes LGBTQ.

\section{CONCLUSION}

Bien que l'on trouve de nombreuses méthodes pour présenter le concept de "queer" ou l'homosexualité dans la société, y compris par l'intermédiaire de films, la fiction autour de la communauté queer les marginalise toujours. Dans le film 120 Battements par Minute (2017), le groupe LGBTQ est au cœur de la fiction. Chaque scène est racontée selon leur point de vue face à la crise de VIH/SIDA. La communauté LGBTQ a fait preuve de courage pour s'exposer dans l'espace public et faire entendre la voix de groupes minoritaires alors que la communauté hétérosexuelle la considère plutôt comme un parasite de la société et les punit.

Le groupe LGBTQ dans cette fiction est perçu comme sujet marginalisé. La marginalisation se concrétise par l'usage de bouc émissaire. Il est au cœur de la narration, mais traité comme un groupe marginal. Ce groupe LGBTQ est accusé d'être responsable de l'épidémie du VIH/SIDA, même s'ils ne sont pas à l'origine la propagation de cette maladie. Ils sont des boucs émissaires de la crise VIH/SIDA s'appuyant sur stéréotypes et des suspects labellisés au sein de leur groupe. A la fin, la société les exclue. Les discours de haine sont reproduits qui montre l'image négative des groups de LGBTQ. Ces discours peuvent facilement trouvé dans les 
choix de mots, les images crées dans le film. Le regards sociaux placent les groups LGBTQ en tant les groups qui sont hors de normes..

\section{REMERCIEMENTS}

Je remercie à tous les professeurs du département $\mathrm{du}$ français d'Universitas Gadjah Mada qui m'ont donné tous les soutiens dont j'ai besoin; les ressources, les idées, et les information sur cette topique. Je remercie notamment à madame Wening UDASMORO, professeur de littérature française et gendre, pour ses enseignements et orientations.

\section{RÉFÉRENCES}

Atwood, C. P. (2009). The Sacrificed Brother in the "Secret History of the Mongols". Mongolian Studies, 30, 189206.

Balsam, K. F. (2011). Measuring Multiple Minority Stress: The LGBT People of Color Microaggressions Scale. Cultural Diversity and Ethnic Minority Psychology, 17(2), 163-174.

Czach, L. (2004). Film Festivals, Programming, and the Building of a National Cinema. The Moving Image, $4(1), 76-88$.

Douglas, T. (2003). Scapegoats: Transfering Blame. London: Taylor and Francis ELibrary.

Girard, R. (1982). Le Bouc Emmisaire. Paris: Grasset \& Fasquelle.

Humbert, D. (2010). Desire and Monstrosity in the Disaster Film: Alfred Hitchcock's "The Birds". Contagion: Journal of Violence, Mimesis, and Culture, 17, 87-103.

Johnston, C. (2002). Representations of homosexuality in 1990s mainstream
French cinema. Studies in French Cinema, 2(1), 23-31.

Leeuwen, T. V. (2004). Introducing Social Semiotics. New York: Routledge.

McGill, A. B. (2018). The Witch, the Goat and the Devil: A Discussion of Scapegoating and the Objectification of Evil in Robert Eggers' The Witch. Theology Today, 74(4), 409-414.

Müller, A. (2017). Beyond 'invisibility': queer intelligibility and symbolic annihilation in healthcare. Culture, Health \& Sexuality, 1-14.

Provencher, D. M. (2008). Tracing Sexual Citizenship and Queerness in Drôle de Félix (2000) and Tarik el hob (2001). Contemporary French and Francophone Studies, 12(1), 51-61.

Rees-Roberts, N. (2008). French Queer Cinema. Edinburgh: Edinburgh University Press.

Rich, B. R. (2000). Queer and present danger: after New Queer Cinema. Retrieved July 10, 2019, from The International Magazine: Sight \& Sound: https://www.bfi.org.uk/newsopinion/sight-soundmagazine/features/queer-presentdanger-b-ruby-rich

Román, D. (1992). 'It's My Party and I'll Die If I Want to!': Gay Men, AIDS, and the Circulation of Camp in U.S. Theatre. Theatre Journal, 44(3), 305-327.

Sindhunata, G. P. (2007). Kambing Hitam: Teori René Girard. Jakarta: Gramedia Pustaka Utama.

Slater, I. (2012). The Last Fetter: A Girardian Reading of Aelred Of Rievaulx's "De Quadam Miraculum Mirabili". Literature and Theology, 26(1), 23-37. 